\title{
DCE-MRI in Glioma, Infiltration Zone and Healthy Brain to Assess Angiogenesis: A Biopsy Study
}

\author{
Vera C. Keil ${ }^{1,2}$ (D) - Gerrit H. Gielen ${ }^{3}$ Bogdan Pintea ${ }^{4,5}$ • Peter Baumgarten ${ }^{6,7}$ - Angeliki Datsi ${ }^{8}$ • \\ Kanishka Hittatiya ${ }^{9} \cdot$ Matthias Simon ${ }^{5,10} \cdot$ Elke Hattingen ${ }^{1,11}$
}

Received: 9 November 2020 / Accepted: 22 March 2021 / Published online: 26 April 2021

(c) The Author(s) 2021

\begin{abstract}
Purpose To explore the focal predictability of vascular growth factor expression and neovascularization using dynamic contrast-enhanced magnetic resonance imaging (DCE-MRI) in glioma.

Methods 120 brain biopsies were taken in vital tumor, infiltration zone and normal brain tissue of 30 glioma patients: $17 \mathrm{IDH}$ (isocitrate dehydrogenase)-wildtype glioblastoma (GBM), $1 \mathrm{IDH}$-wildtype astrocytoma ${ }^{\circ} \mathrm{III}$ (together prognostic group 1), 3 IDH-mutated GBM (group 2), 3 anaplastic astrocytomas IDH-mutated (group 3), 4 anaplastic oligodendrogliomas and 2 low-grade oligodendrogliomas (together prognostic group 4). A mixed linear model evaluated the predictabilities of microvessel density (MVD), vascular area ratio (VAR), mean vessel size (MVS), vascular endothelial growth factor and receptors (VEGF-A, VEGFR-2) and vascular endothelial-protein tyrosine phosphatase (VE-PTP) expression from Tofts model kinetic and model-free curve parameters.

Results All kinetic parameters were associated with VEGF-A (all $p<0.001)$ expression. $\mathrm{K}^{\text {trans }}, \mathrm{k}_{\mathrm{ep}}$ and $\mathrm{v}_{\mathrm{e}}$ were associated with VAR ( $p=0.006,0.004$ and 0.01 , respectively) and MVS ( $p=0.0001,0.02$ and 0.003 , respectively) but not MVD $\left(p=0.84,0.74\right.$ and 0.73 , respectively). Prognostic groups differed in $\mathrm{K}^{\text {trans }}(p=0.007)$ and $\mathrm{v}_{\mathrm{e}}(p=0.004)$ values measured in the infiltration zone. Despite significant differences of VAR, MVS, VEGF-A, VEGFR-2, and VE-PTP in vital tumor tissue and the infiltration zone ( $p=0.0001$ for all), there was no significant difference between kinetic parameters measured in these zones.

Conclusion The DCE-MRI kinetic parameters show correlations with microvascular parameters in vital tissue and also reveal blood-brain barrier abnormalities in the infiltration zones adequate to differentiate glioma prognostic groups.
\end{abstract}

All coauthors consented to manuscript submission in its present form.

Availability of data and material The datasets generated during and/or analyzed during the current study are available from the corresponding author on reasonable request.

Code availability Not applicable.

Vera C. Keil

v.c.w.keil@amsterdamumc.nl

1 Department of Neuroradiology, University Hospital Bonn, Venusberg-Campus 1, 53127 Bonn, Germany

2 Department of Radiology, Amsterdam University Medical Center, location VUmc, De Boelelaan 1117, 1081 HV Amsterdam, The Netherlands

3 Department of Neuropathology, University Hospital Bonn, Venusberg-Campus 1, 53127 Bonn, Germany

4 Department of Neurosurgery, University Hospital BG Bergmannsheil, Bürkle-de-la-Camp-Platz 1, 44789 Bochum, Germany
5 Department of Neurosurgery, University Hospital Bonn, Venusberg-Campus 1, 53127 Bonn, Germany

6 Department of Neurosurgery, University Hospital Frankfurt, Schleusenweg 2-16, 60528 Frankfurt am Main, Germany

7 Institute of Neuropathology (Edinger Institute), University Hospital Frankfurt, Schleusenweg 2-16, 60528 Frankfurt am Main, Germany

8 ITZ, Heinrich-Heine-University Düsseldorf, Moorenstraße 5, 40225 Düsseldorf, Germany

9 Center for Pathology, University Hospital Bonn, Venusberg-Campus 1, 53127 Bonn, Germany

10 Department of Neurosurgery, Ev. Krankenhaus Bielefeld, Haus Gilead I, Burgsteig 13, 33617 Bielefeld, Germany

11 Department of Neuroradiology, University Hospital Frankfurt, Schleusenweg 2-16, 60528 Frankfurt am Main, Germany 
Keywords Glioma $\cdot$ Biopsy VEGF $\cdot$ Blood-brain barrier $\cdot$ Multiparametric magnetic resonance imaging

\section{Introduction}

Glioma vessels show multilevel abnormalities with hyperperfusion, a variable blood-brain barrier disruption (BBBD) and pathological vessel sizes and densities. Vascular endothelial growth factor (VEGF) driven angiogenesis is directly related to lesion malignancy and potential treatment susceptibility, lesion expansion, and secondary tumor dedifferentiation [1-3]. Monitoring microvessel status, including $\mathrm{BBBD}$ and the evolution of proangiogenic proteins, can be an essential feature to control the efficacy of glioma treatment [4].

Glioma vascularization can be assessed on a molecular level, e.g. through the analysis of expression of vascular endothelial growth factor (VEGF) the VEGF receptors (VEGFR) or vascular endothelial-protein tyrosine phosphatase (VE-PTP), which is associated with BBBD, as well as histologically studying microvessel density or presence of enlarged vessels (ESM 1) [5]. Gliomas are intraindividually heterogeneous $[6,7]$ and noninvasive methods are necessary to evaluate vascular proliferations to facilitate optimization of surgical resection planning. Magnetic resonance imaging (MRI) studies using dynamic susceptibility contrast MRI (DSC-MRI) delineated several vascular zones, or habitats, of distinct perfusion within a tumor. These have a prognostic relevance and extend beyond the glioma's outer margins into the infiltration zone [8-10].

Dynamic contrast-enhanced MRI (DCE-MRI) is designed to provide a quantitative measure for BBBD [11, 12]. Its kinetic parameters contrast agent efflux transfer constant $\left(\mathrm{K}^{\text {trans }}\right)$, contrast agent reflux transfer constant $\left(\mathrm{k}_{\mathrm{ep}}\right)$, the extracellular-extravascular volume fraction $\left(\mathrm{v}_{\mathrm{e}}\right)$ and the intravascular volume fraction $\left(\mathrm{v}_{\mathrm{p}}\right)$, a suspected correlate for vascular space, were shown to correlate with glioma WHO grades, genomic prognostic markers, microvascular anatomy such as vessel density, as well as VEGF expression [2, 3, 7, 13-18]. Kinetic parameters are influenced by both vessel perfusion and permeability and hence may be particularly useful to serve as imaging markers for vascular proliferation in gliomas (ESM 1). The reliability of DCE-MRI regarding the focal assessment of vascular parameters is, however, poorly examined [19]. This uncertainty includes the glioma infiltration zone, which only microscopically shows subtle tumor cell infiltration, especially in normal-appearing brain tissue $[2,20$, 21]. Treatment planning and evaluation in patients with glioma must consider these peripheral regions. A meticulous confirmation of MRI kinetic parameters measurements is necessary to support the strength of MRI as a source of noninvasive biomarkers for vascular conditions in the brain. On the other hand, experimental DCE-MRI protocols showed enough sensitivity to detect very subtle BBBD in dementia patients [22]. The difficult aspect of a DCEMRI study that involves analyses of lesions with a high permeability and perfusion next to lesions with variable or no permeability is that the kinetic models are not the same for all tissues. Not even the acquisition parameters of the dynamic sequence are optimal for all tissues. Highly perfused and permeable tumors will most likely be reliably assessed using the extended Tofts model, while lesions with low to no BBBD should be assessed with models sensitive to low BBBD, such as the Patlak model, or be analyzed model-free altogether [23]. There will thus be a trade-off in reliability or comparability, depending on whether one or several models are used to analyze these different tissue zones.

In order to advance the clinical implementation of DCEMRI as a noninvasive method to evaluate glioma vascularization, this study aimed to explore if DCE-MRI kinetic parameters can focally predict histological and molecular vascular parameters in different vascular zones of and beyond glioma tissue and how this is related to the histology and expected prognosis of the lesion.

\section{Material and Methods}

\section{Study Overview and Cohort Selection}

This prospective study was approved by the university ethics committee (study approval number 358/13). Adult patients participated after written informed consent. Inclusion criteria were: 1) suspected or previously histologically confirmed glioma, 2) no contraindications against MRI, and 3) planned surgery. DCE-MRI was added to the standard preoperative MRI. Tissue samples covered vital tissue, the infiltration zone and visually healthy brain in areas needing to be resected anyhow. Tissue was diagnosed according to the 2016 WHO classification for central nervous system (CNS) tumors and analyzed for microvascular parameters, VEGF-A, VEGFR-2 and VE-PTP expression [24].

\section{MRI Acquisition and Processing}

All patients were examined at 3.0T (Achieva TX, Philips Healthcare, Best, The Netherlands) using an 8-channel head coil. The 3D contrast-enhanced T1-weighted sequence was 
acquired for intraoperative neuronavigation (TR $7.5 \mathrm{msec}$, TE $3.5 \mathrm{msec}$; FA $8^{\circ}$, FOV $256 \times 256 \times 256 \mathrm{~mm}$, slices 170 , scan time $4 \mathrm{~min} 40 \mathrm{sec}$, voxel size $1.0 \times 1.0 \times 1.0 \mathrm{~mm}^{3}$ ). The DCE-MRI sequence involved a double flip-angle technique and 50 dynamic scans (whole-brain coverage, FOV $108 \times 220 \times 182 \mathrm{~mm}, 36$ slices, voxel size $1.57 \times 1.6 \times 3 \mathrm{~mm}$, scan time $5 \mathrm{~min} 51 \mathrm{sec}$; T1 reference scans: TR $10 \mathrm{msec}$, TE $2.3 \mathrm{msec}$, flip angles $5 / 15^{\circ}$; dynamic scans $(5.3 \mathrm{sec}$ each): TR $3.5 \mathrm{msec}$, TE $2.3 \mathrm{msec}$, flip angle $8^{\circ}$ ). Gadobutrol (Gadovist ${ }^{\circledR}$, Bayer Healthcare, Leverkusen, Germany; standard dose $0.1 \mathrm{mmol} / \mathrm{kg}$ body weight) was the contrast agent injected at $3 \mathrm{~mL} / \mathrm{s}$ through an antecubital vein followed by $25 \mathrm{~mL}$ flush saline. The assumed relaxivity at $37{ }^{\circ} \mathrm{C}$ body temperature was $5.0 /\left(\mathrm{mmol}^{*} \mathrm{~s}\right)$. Kinetic parameters were calculated based on the extended Tofts model with an arterial input function derived from individually placed regions of interest (ROI) in the superior sagittal sinus (Intellispace Portal 5.0, Philips Healthcare, Best, The Netherlands). Choosing the extended Tofts model for all ROI including healthy brain is a compromise to maintain comparability of the data. An additional model-free analysis of the area under the receiver operating characteristic curve (AUC) and the maximum relative enhancement $(\mathrm{mrE})$ were an approach to compensate for this circumstance. The $\mathrm{mrE}$ is defined as the maximum signal difference compared to baseline in percent (\%).

Kinetic parameter maps were fused with the 3D neuronavigation datasets to be used for ROI placement. To measure kinetic parameters, a neuroradiologist (V.C.K.) selected $0.5 \times 0.5 \times 0.5 \mathrm{~cm}^{3}$ ROI in the following zones (Fig. 1):
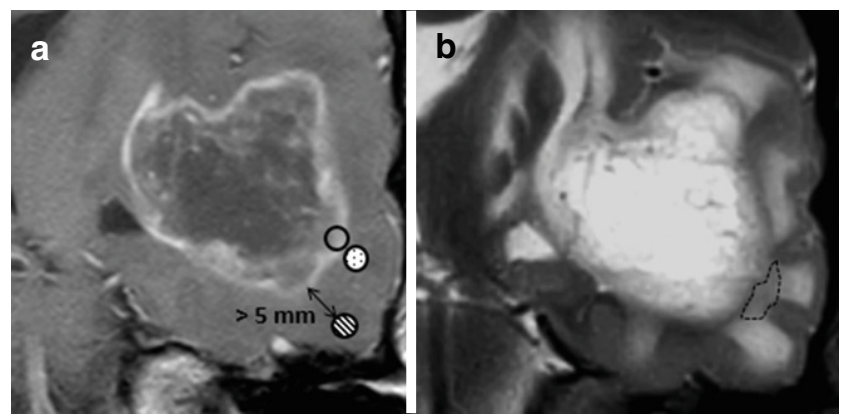

Fig. 1 Tissue zones in glioblastoma. a Partial coronal view of a left temporal glioblastoma in a 56-year-old man (T1-weighted plus contrast). The clear black circle marks vital glioma tissue (zone 1). The adjacent dotted circle marks the infiltration zone (zone 2). The striped circle defined the suspected zone 3 (visually healthy tissue) in a minimum distance of $5 \mathrm{~mm}$ to the enhancing glioma rim. $\mathbf{b}$ The corresponding T2-weighted image serves as a radiological verification of glioma zones with zone 2 being in a T2 hypointense area (striped outline), while zone 3 represents normal cortex and subcortical mild vasogenic edema. N.B. tissue had to appear healthy intraoperatively under the microscope
- Zone 1: contrast-enhancing glioma and/or T2-hyperintense tissue,

- Zone 2: suspected infiltration zone,

- Zone 3: visually healthy brain tissue with more than $5 \mathrm{~mm}$ distance to the outer contrast-enhancing area of the tumor with either high signal on $\mathrm{T} 2$ or brain-isointense signal.

The total number of planned biopsies varied by tumor size and safe resectability of peripheral tissue in zones 2 and 3 from 2-6 biopsies per patient.

\section{Tissue Preparation and Analyses}

Kinetic parameter corresponding biopsies were identified by pointer navigation in Softtouch ${ }^{\circledR}$ referencing technique (M.S. and B.P., Brainlab AG, Munich, Germany) based on the marked ROI and were all successfully retrieved at the beginning of surgery to minimize brain shift effects. They were formalin-fixed and paraffin-embedded. Routine neuropathological diagnostics to achieve a diagnosis according to the revised 4th edition of the 2016 WHO classification of tumors of the CNS was performed on independently taken biopsy tissue (G.H.G.) [24]. Based on the publication by Hartmann et al. four prognostic groups were created out of all patients' WHO 2016 diagnoses: 1) short survival: glioblastoma ${ }^{\circ} \mathrm{IV}$ and astrocytoma ${ }^{\circ} \mathrm{III}$, both IDH-wildtype; 2) shorter intermediate survival: glioblastoma ${ }^{\circ} \mathrm{IV}$, IDH-mutated; 3) longer intermediate survival: astrocytoma ${ }^{\circ} \mathrm{III}$, IDH-mutated and 4) longer survival: oligodendroglioma ${ }^{\circ} \mathrm{III}$ and ${ }^{\circ} \mathrm{II}$, IDHmutated, $1 \mathrm{p} / 19 \mathrm{q}$ co-deleted [25]. In prognostic group 1 , zone 1 biopsies, which-different from preoperative expectation on MRI-histologically identified as mainly necrotic were only analyzed concerning descriptive statistics of the respective microvascular and kinetic parameters, but were not considered in prognostic group or zone analyses.

For microvessel analysis, $4 \mu \mathrm{m}$ thick tissue specimens were stained against endothelial marker CD34 (Ventana Benchmark XT Immunostainer, Roche Ventana, Darmstadt, Germany; 1/100 antibody: DAKO, Glostrup, Denmark). Digital scans of the tissue slides were analyzed with quantification software (Tissue Studio ${ }^{\circledR}$, Definiens, Munich, Germany). Automated settings were subjected to manual training adjustments in order to exclude artefacts (K.H.). Microvessels were defined as vessel areas smaller than $2.0 \mathrm{~mm}^{2}$. The software defined microvascular parameters based on $0.6 \times 0.6 \mathrm{~mm}^{2}$ target squares and $\mathrm{t} 3$ microvascular parameters (vascular area ratio, VAR, microvessel density, MVD, mean microvessel size, MVS) were determined. To calculate the VAR, which is the percentage of area covered by blood vessels in comparison to the area covered by other tissues on the slide, all single areas of vascular tissue 
(including their lumina) were added and divided by the remaining nonvascular tissue area. Vascularization data of all analyzed tiles in a biopsy section were averaged to the mean.

For VE-PTP, $4 \mu \mathrm{m}$ thick tissue sections were stained with purified mouse anti-human VEGF-A (clone G153694) and VE-PTP (BD Pharmingen, Heidelberg, Germany; clone 12; indirect immunohistochemistry). Antigen retrieval was achieved using $90^{\circ} \mathrm{C}$ target retrieval solution (DAKO, Glostrup, Denmark) and washing in phosphate buffered saline with $0.3 \%$ Triton X-100 (Sigma Aldrich, Steinheim, Germany). Primary antibody (VE-PTP 1:150) was diluted in $1 \%$ bovine serum antigen and stained at $4{ }^{\circ} \mathrm{C}$. Secondary $1 / 500$ biotinylated goat anti-mouse-antibody (DAKO, Glostrup, Denmark) was applied for $45 \mathrm{~min}$ at room temperature. Amplification involved the ABC-(HRP)kit (VECTA Laboratories, Peterborough, UK) and 3,3'-diaminobenzidine was the chromogenic substrate (VECTA Laboratories, Peterborough, UK). IgG-negative controls were run. Free VE-PTP expression was assessed by the scoring system of Raica et al. [26]. Intensity and percentage of VE-PTP positive stained cells were analyzed.

Tumor sections $(4 \mu \mathrm{m})$ were subjected to immunohistochemistry for VEGF-A and VEGFR-2 using mouse IgG2b anti-human VEGF (clone: MAB293, dilution 1:100; R\&D Systems, Minneapolis, MN, USA) and monoclonal rabbit anti-human VEGFR-2 dilution 1:500 (clone: 55B11; Cell Signaling, Danvers, MA, USA). Tissue labeling was performed using the Leica Bond III immunohistochemistry system (Leica Biosystems, Buffalo Grove, IL, USA). For VEGF-A, a cell conditioning pretreatment with enzyme was performed for $10 \mathrm{~min}$, for VEGFR-2 cell conditioning was performed with EDTA for $20 \mathrm{~min}$. The primary antibodies were applied for $15 \mathrm{~min}$. Standard secondary antibody (Leica Biosystems) was incubated for $8 \mathrm{~min}$. For diaminobenzidine (DAB) visualization and counterstaining, the Bond Polymer Refine Detection Kit (Leica Biosystems) was used. High overlap with mRNA levels demonstrated by in situ hybridization for the soluble VEGF-A could be proven [27, 28].

For quantification of VEGF-A and VEGFR-2 expression, a semiquantitative score was used. The immunohistochemical staining intensity (negative $=0$, low $=1$, moderate $=2$, strong $=3$ ) was multiplied by the proportion of positive tumor or vascular cells separately. Each product was then added to each other receiving a sum score. This histological score is well evaluated as published before [29, 30].

\section{Statistics}

Statistical analyses were performed with SPSS 24.0 (IBM, Armonk, NY, USA). First, $\mathrm{K}^{\text {trans }}$ kinetic parameter results were Pearson correlated with AUC and mrE of the sig- nal intensity time curves to explore if model-free measurements, which are possibly less affected by false results due to low permeability than kinetic parameters of the Tofts model, are related to kinetic parameters. This was taken as an indirect corroboration of the reliability of the kinetic parameters. The operation was performed for all tissue zones separately. The DCE-MRI kinetic parameters were explored regarding their capacity to predict microvascular parameters, VEGF-A, VEGFR-2 and VE-PTP scores in a generalized linear (dichotomous data) or linear mixed (continuous data) model approach with the patient as the random factor. A linear mixed model similarly determined tissue zone-dependent differences of parameters. KruskalWallis tests were used to identify parameter differences between prognostic groups measured in the same zones. The ROC analyses determined the discriminability tissue zones or prognostic groups by kinetic parameters. Analyses were performed with inclusion of recurrent cases after evaluation of significant parameter differences (Whitney-Mann U-test stratified by tumor zones and prognostic groups) in these groups to rule out possible effects of chemoradiotherapy. Multiple testing correction for linear mixed models is difficult. All other results are also stated after Bonferroni correction.

\section{Results}

\section{Cohort Demographics, Biopsy Overview and Parameter Correlations}

Patients and diagnoses as well as biopsy numbers are listed in ESM 2. An overview of the biopsies, the histology and tissue zones, is presented in Tables 1 and 2. An example of tissue zones in one individual is given in ESM 3. Note that tissue presumed to lie in zone 2 or 3 indeed was later rated as infiltration zone or normal brain in all cases, while 14 out of 68 planned biopsies in vital tumor turned out to be mainly necrotic tissue reducing the amount of analyzed zone 1 to 3 biopsies to 106 (all in IDH-wildtype GBM, Tables 1 and 2). The mean kinetic parameter values and model-free values are presented in ESM 4.

The correlation coefficient for $\mathrm{K}^{\text {trans }}$ with the AUC was $0.78(p<0.0001)$ and $0.85(p<0.0001 ; n=106$ biopsies/ROI $)$ with the $\mathrm{mrE}$ when considering all biopsies. Taking only zones 2 and 3 ROI into account these values were 0.92 and 0.90 , respectively $(n=52)$.

\section{Relationship of Kinetic MRI Parameters with Vascular Parameters}

All cases $(n=30,106$ biopsies) were considered in this analysis due to a lack of significant parameter value differ- 
Table 1 Biopsies and diagnoses

\begin{tabular}{|c|c|c|c|}
\hline \multicolumn{4}{|c|}{ WHO 2016 diagnosis including tumor grades } \\
\hline- & $\begin{array}{l}\text { Number of patients and } \\
\text { biopsies }\end{array}$ & Prognostic groups according to WHO 2016 & $\begin{array}{l}\text { Number of patients and } \\
\text { biopsies }\end{array}$ \\
\hline $\mathrm{GBM}^{\circ} \mathrm{IV}$ IDHwt & $\begin{array}{l}n=17 \\
\text { ( } 71 \text { biopsies) }\end{array}$ & $\begin{array}{l}\text { (1) short survival: } \\
\text { GBM }{ }^{\circ} \text { IV and Astrocytoma }{ }^{\circ} \text { III, IDHwt }\end{array}$ & $\begin{array}{l}n=18 \\
\text { ( } 75 \text { biopsies) }\end{array}$ \\
\hline $\begin{array}{l}\text { Astrocytoma }{ }^{\circ} \mathrm{III}, \\
\text { IDHwt }\end{array}$ & $\begin{array}{l}n=1 \\
\text { ( } 4 \text { biopsies) }\end{array}$ & & \\
\hline $\mathrm{GBM}^{\circ} \mathrm{IV}, \mathrm{IDHmut}$ & $\begin{array}{l}n=3 \\
(15 \text { biopsies })\end{array}$ & $\begin{array}{l}\text { (2) shorter intermediate survival: } \\
\text { GBM }{ }^{\circ} \mathrm{IV} \text {, IDHmut }\end{array}$ & $\begin{array}{l}n=3 \\
(15 \text { biopsies })\end{array}$ \\
\hline $\begin{array}{l}\text { Astrocytoma }{ }^{\circ} \mathrm{III}, \\
\text { IDHmut }\end{array}$ & $\begin{array}{l}n=3 \\
\text { ( } 8 \text { biopsies })\end{array}$ & $\begin{array}{l}\text { (3) longer intermediate survival: } \\
\text { Astrocytoma }{ }^{\circ} \mathrm{III}, \mathrm{IDH} \text { ut }\end{array}$ & $\begin{array}{l}n=3 \\
(8 \text { biopsies) }\end{array}$ \\
\hline $\mathrm{ODG}^{\circ} \mathrm{III}, \mathrm{IDHmut}$ & $\begin{array}{l}n=4 \\
\text { (16 biopsies) }\end{array}$ & $\begin{array}{l}\text { (4) longer survival: } \\
\mathrm{ODG}^{\circ} \mathrm{III} \text { and }{ }^{\circ} \mathrm{II}, \mathrm{IDHmut}\end{array}$ & $\begin{array}{l}n=6 \\
(22 \text { biopsies })\end{array}$ \\
\hline $\mathrm{ODG}^{\circ} \mathrm{II}, \mathrm{IDHmut}$ & $\begin{array}{l}n=2 \\
(6 \text { biopsies })\end{array}$ & & \\
\hline \multicolumn{4}{|c|}{ Number of biopsies by WHO 2016 grades } \\
\hline${ }^{\circ} \mathrm{IV}$ & 86 & - & \\
\hline${ }^{\circ} \mathrm{III}$ & 28 & - & \\
\hline${ }^{\circ} \mathrm{II}$ & 6 & - & \\
\hline
\end{tabular}

$n=30$ patients ( 9 women, 38 biopsies; 21 men, 82 biopsies)

Total biopsies $n=120$ ( $n=94$ de novo tumors, $n=26$ recurrent cases)

GBM glioblastoma, IDHwt/mut isocitrate dehydrogenase wildtype/mutated (or mutant), $O D G$ oligodendroglioma, WHO World Health Organization

Table 2 Biopsy zone distribution by histopathological confirmation

\begin{tabular}{|c|c|c|c|c|c|}
\hline & \multirow[t]{2}{*}{ Total } & \multicolumn{4}{|c|}{ By WHO 2016 prognostic group } \\
\hline & & 1 & 2 & 3 & 4 \\
\hline Necrosis & $\begin{array}{l}14 \\
\text { biopsies }^{\mathrm{a}}\end{array}$ & $14^{\mathrm{a}}$ & - & - & - \\
\hline $\begin{array}{l}\text { Vital tumor } \\
\text { (zone 1) }\end{array}$ & $\begin{array}{l}54 \text { biop- } \\
\text { sies }\end{array}$ & 35 & 8 & 4 & 7 \\
\hline $\begin{array}{l}\text { Infiltration zone } \\
\text { (zone 2) }\end{array}$ & $\begin{array}{l}33 \text { biop- } \\
\text { sies }\end{array}$ & 15 & 4 & 3 & 11 \\
\hline $\begin{array}{l}\text { Normal brain } \\
\text { (zone 3) }\end{array}$ & $\begin{array}{l}19 \text { biop- } \\
\text { sies }\end{array}$ & 11 & 3 & 1 & 4 \\
\hline
\end{tabular}

$n=30$ patients (9 women, 38 biopsies; 21 men, 82 biopsies)

Total biopsies $n=120$ ( $n=94$ de novo tumors, $n=26$ recurrent cases)

WHO World Health Organization

${ }^{a}$ not used in further comparative zone analyses being a necrotic biopsy

ences between de novo and recurrent cases. The $\mathrm{K}^{\text {trans }}, \mathrm{k}_{\mathrm{ep}}$ and $\mathrm{v}_{\mathrm{e}}$ were significantly associated with VAR $(p=0.006$, 0.004 and 0.01 , respectively) and MVS ( $p=0.0001,0.02$ and 0.003 , respectively), but not MVD ( $p=0.84,0.74$ and 0.73 , respectively). The $v_{p}$ was only significantly associated with MVS $(p=0.002)$, but not VAR $(p=0.09)$ or MVD $(p=0.93)$.

Looking at biopsies including all tissue zones $(n=106$ biopsies), all kinetic parameters were significantly associated with VEGF-A expression (all $p<0.001$ ), while VEGFR-2 expression did not reach a significant association with $\mathrm{K}^{\text {trans }}, \mathrm{v}_{\mathrm{e}}$ or $\mathrm{v}_{\mathrm{p}}(p=0.08,0.13$ and 0.15 respectively), but with $\mathrm{k}_{\mathrm{ep}}(p=0.04)$ only.
There was no association between any of the kinetic parameters with VE-PTP expression ( $\mathrm{K}^{\text {trans }} p=0.13, \mathrm{k}_{\mathrm{ep}}$ $p=0.12, \mathrm{v}_{\mathrm{e}} p=0.44$ and $\mathrm{v}_{\mathrm{p}} p=0.27$ ).

\section{Relevance and Identification of Tissue Zones Regarding Kinetic and Microvascular Parameters}

The detailed analysis split by single tissue zones (compare Tables 1 and 2) showed that the association between kinetic parameters and vascular parameters was largely based on the vital glioma tissue, and that $\mathrm{K}^{\text {trans }}$ was the most reliably correlated kinetic parameter (Fig. 2); however, VEGF-A showed an association with more kinetic parameters in the infiltration zone rather than in vital tumor tissue (Fig. 2).

Regarding the differentiability of vital tumor from the infiltration zone in gliomas of the same prognostic group (compare Tables 1 and 2$)$, VAR $(2.9 \pm 0.4$ vital tumor vs. $1.1 \pm 0.1$ infiltration zone vs. $1.3 \pm 0.1$ normal brain, in $\%)$, MVS (387.1 \pm 27.5 vital tumor vs. $193.5 \pm 15.6$ infiltration zone vs. $178.3 \pm 17.5$ normal brain) as well as VEGF-A $(42.3 \pm 9.8$ vital tumor vs. $9.5 \pm 7.2$ infiltration zone vs. $0.3 \pm 0.3$ normal brain), VEGFR-2 $(108.7 \pm 14.3$ vital tumor vs. $18.3 \pm 9.3$ infiltration zone vs. $4.3 \pm 2.0$ normal brain) and VE-PTP $(0.5 \pm 0.1$ vital tumor vs. $0.1 \pm 0.1$ infiltration zone vs. 0 normal brain) scores were all significantly higher in vital tumor tissue than in the infiltration zones or normal brain ( $p=0.0001$ for all; mixed linear model); however, kinetic parameters did not differ significantly between zones (ESM 4). 
a

Vital glioma tissue Kinetic parameters

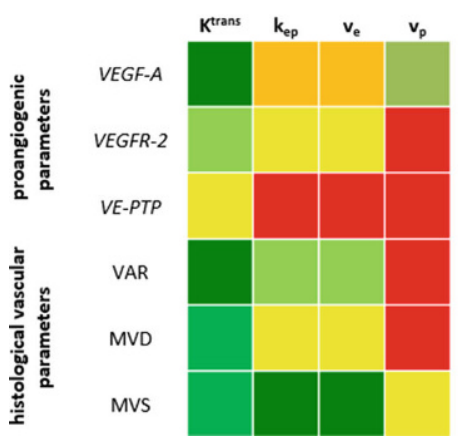

b



\section{Infiltration zone}

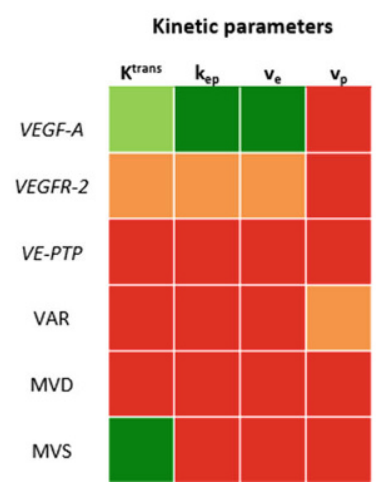

C

Healthy tissue

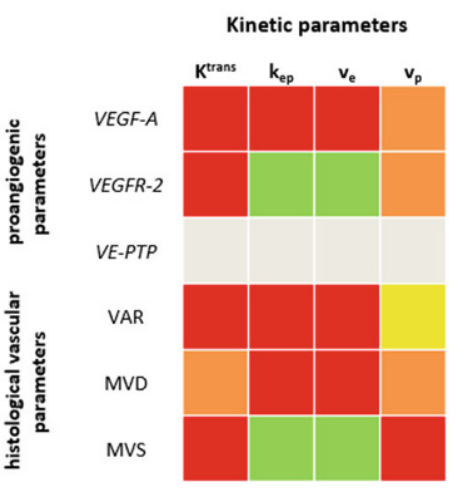

Color coding of p-values

\begin{tabular}{|c|}
\hline $1-0.5$ \\
\hline$<0.5-0.1$ \\
\hline$<0.1-0.05$ \\
\hline$<0.05-0.01$ \\
\hline$<0.01-0.001$ \\
\hline$<0.001$ \\
\hline
\end{tabular}

Fig. 2 Heat map presentation of correlations between kinetic and vascular parameters. a Vital glioma tissue zone 1, b infiltration zone 2 , $\mathbf{c}$ healthy appearing tissue zone 3. N.B. no statistics could be performed for VE-PTP in healthy appearing tissue as all samples showed the same nonexpression (score 0 , expressed in grey)
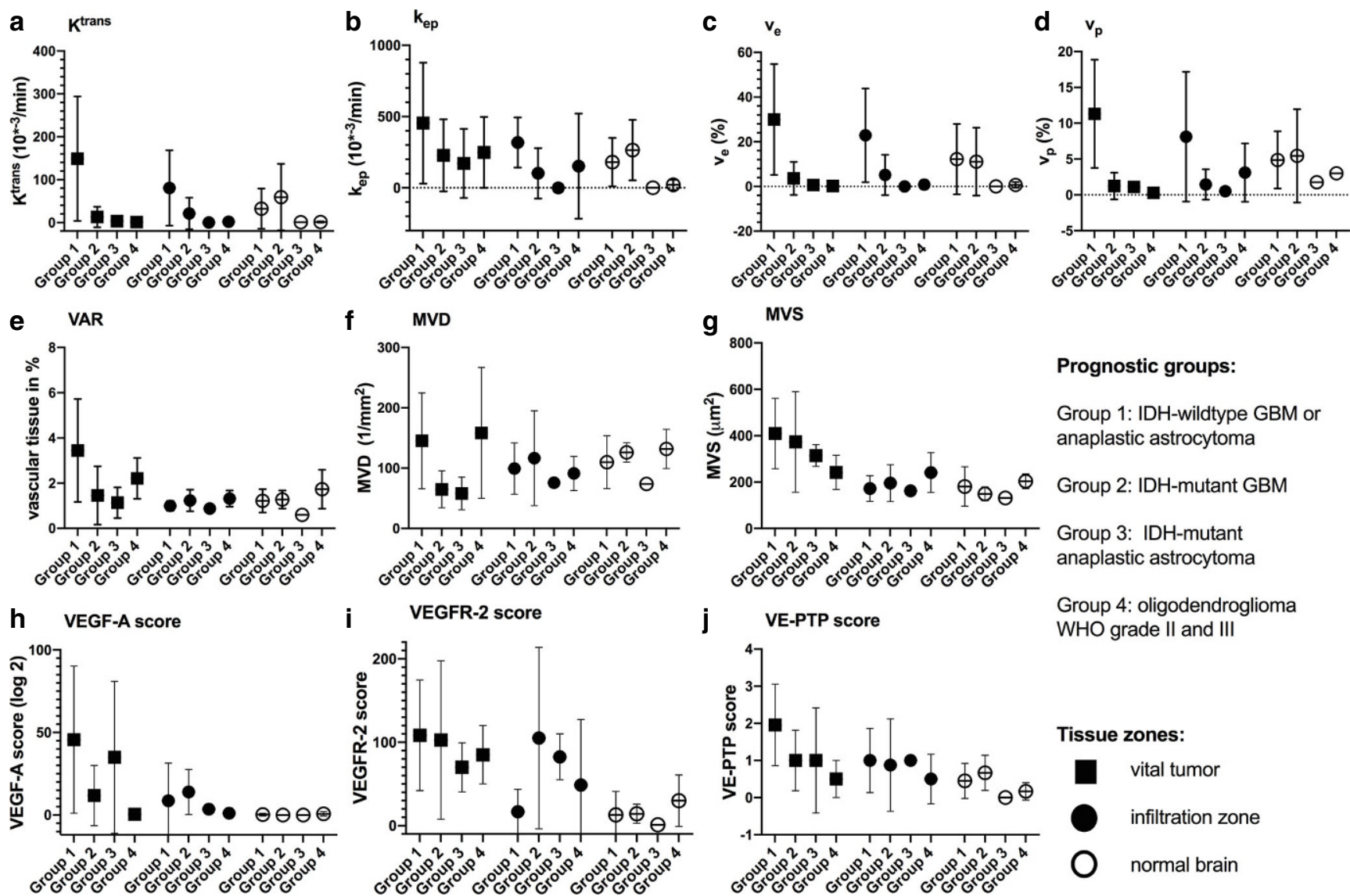

Prognostic groups:

Group 1: IDH-wildtype GBM or anaplastic astrocytoma

Group 2: IDH-mutant GBM

Group 3: IDH-mutant anaplastic astrocytoma

Group 4: oligodendroglioma WHO grade II and III

Tissue zones:

vital tumor

infiltration zone

normal brain

Fig. 3 Differences of kinetic and vascular parameters between tissue zones and prognostic groups. a-d Kinetic parameters, e-g histological microvascular parameters, h-k vascular growth factors (group 1 short survival, glioblastoma ${ }^{\circ} \mathrm{IV}$, IDH-wildtype, group 2 shorter intermediate survival, glioblastoma ${ }^{\circ} \mathrm{IV}$, IDH-mutant, group 3 longer intermediate survival, astrocytoma ${ }^{\circ} \mathrm{III}$, IDH-mutated, group 4 longer survival, oligodendroglioma ${ }^{\circ} \mathrm{III}$ and ${ }^{\circ} \mathrm{II}$, IDH-mutant, $1 \mathrm{p} / 19 \mathrm{q}$ co-deleted, $K^{\text {trans }}$ contrast agent transfer constant (efflux to EES), $k_{e p}$ contrast agent transfer constant (reflux to vessels), $v_{e}$ EES volume fraction (proposed cellularity marker), $v_{p}$ plasma volume fraction (proposed marker for vascularization), $V A R$ vascular area ratio, $M V D$ mean vessel density, $M V S$ mean vessel size, $V E G F-A$ vascular endothelial growth factor type A, VEGFR-2 vascular endothelial growth factor receptor type 2, VE-PTP vascular endothelial-protein tyrosine phosphatase) 
Fig. 3 presents the detailed tissue zones and prognostic group dependent parameter value distribution.

\section{Prognostic Tumor Groups Regarding Kinetic and Microvascular Parameters and Different Tissue Zones}

Comparing vital tumor (zone 1) between the four prognostic tumor groups (compare Tables 1 and 2) $\mathrm{K}^{\text {trans }}(p=0.001), \mathrm{v}_{\mathrm{e}}$ $(p=0.002)$ and $\mathrm{v}_{\mathrm{p}}(p=0.0001)$ differed significantly, but not $\mathrm{k}_{\mathrm{ep}}(p=0.337)$. The $\mathrm{K}^{\text {trans }}$ measured in vital tumor zone 1 was a suitable parameter to differentiate prognostic group 1 from group 2 (AUC $=0.88$, CI 0.76-1.0, $p=0.0009$; Fig. 4). The $\operatorname{VAR}(p=0.008)$ and the MVD $(p=0.009)$ in zone 1 also differed between prognostic groups. This significance was, however, based on the large difference of one prognostic group differing more than the others (Fig. 3). Comparing measurements in zone 1, groups $2-4$ did not significantly differ between each other in any of the parameters including VEGF-A, VEGFR-2 and VE-PTP (Fig. 3 and ESM 4 for kinetic parameters).

The $\mathrm{K}^{\text {trans }}(p=0.007), \mathrm{k}_{\text {ep }}(p=0.019)$ and $\mathrm{v}_{\mathrm{e}}(p=0.004)$ measured in the infiltration zone were significantly different between prognostic groups due to higher kinetic parameter values again in prognostic group 1 (ESM 4). In the infiltration zone, prognostic groups 1 and 2 could be differentiated to some degree (AUC $=0.81$, CI $0.52-1.0, p=0.05$ ) using $\mathrm{K}^{\text {trans }}$. No differences in kinetic parameters were noted when comparing between prognostic groups in normal brain despite higher mean $\mathrm{K}^{\text {trans }}$ and $\mathrm{k}_{\mathrm{ep}}$ in prognostic groups 1 and 2 than in groups 3 and 4.

\section{Discussion}

This interdisciplinary glioma study suggests that vascular growth factors, microvascular anatomy, and DCE kinetic parameters show significant correlations not only in vital tumor tissue, but also in the histologically confirmed infiltration zone of gliomas, albeit to a lesser degree. Second, kinetic parameters and histological microvascular parameters also differed between the group of gliomas with the worst prognosis (glioblastoma, IDH-wildtype) and all other groups when comparing values measured in the infiltration zones of this group versus the others. Finally, VEGF-A showed a broader association with kinetic parameters in tissue of the infiltration zone rather than in the vital tumor tissue.

Substantial percentages of high-grade gliomas do not show avid contrast enhancement [31]. The exchange of contrast agent between the extravascular and intravascular spaces is a multifactorial process influenced by the BBB, quantitative and qualitative aspects of the microvascular network and cerebral blood flow (i.e., perfusion; ESM 1) [32]. One of our hypotheses was that BBBD could be estimated directly by the presence of free vascular endothelialprotein tyrosine phosphatase (VE-PTP), which dissociates from tight junction re-enforcing protein VE-cadherin. Consequently, tight junctions between endothelial cells open and vessels become permeable [5], which would be reflected by DCE-MRI kinetic parameters; however, we could not identify any association between VE-PTP expression and any of the kinetic parameters. While anti-VEGF drug effects seem to be reliably observable using DCE-MRI [3], a

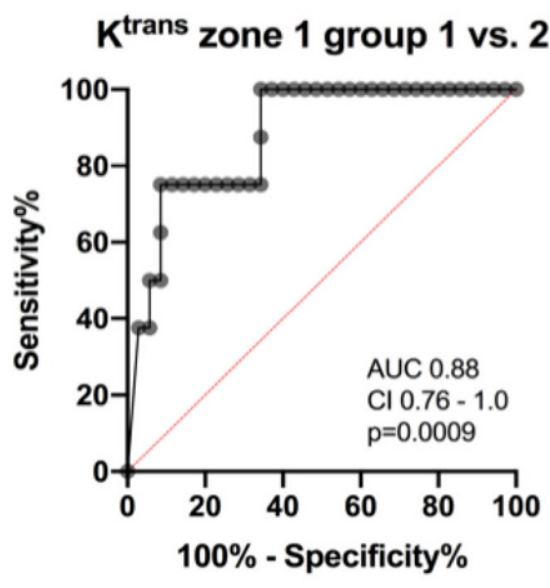

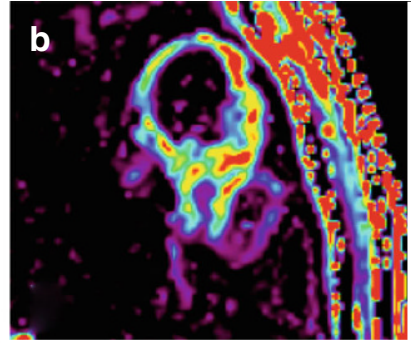
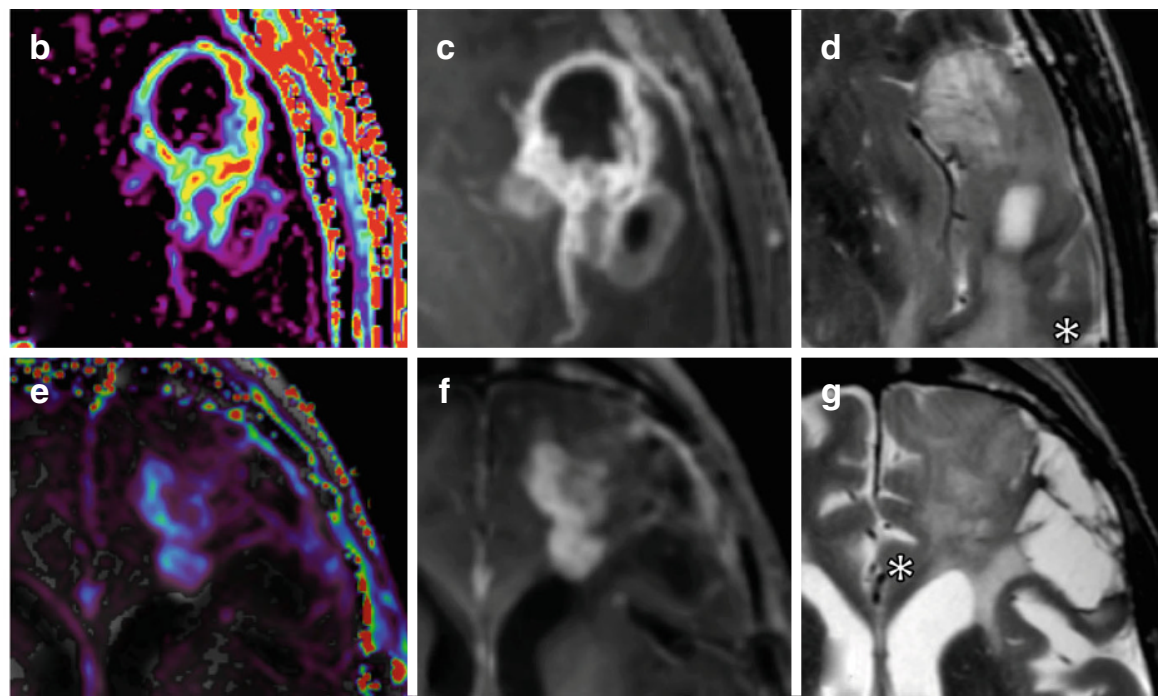

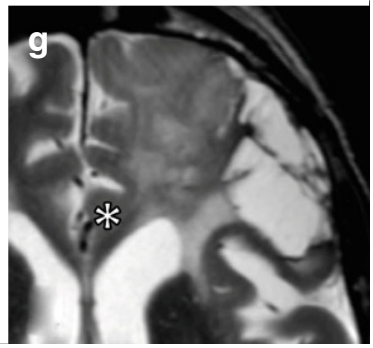

Fig. 4 Example of the prognostic group differentiation potential of $\mathrm{K}^{\text {trans }}$ measured in vital tumor of groups 1 and 2 . a ROC curve, $\mathbf{b}-\mathbf{d}$ example of a 56-year-old patient in prognostic group 1 with a GBM, IDH-wildtype, e-g example of a 77-year-old patient in prognostic group 2 with a GBM, IDH-mutant, b, e $\mathrm{K}^{\text {trans }}$ map, $\mathbf{c}, \mathbf{f}$ contrast-enhanced T1-weighted, d, $\mathbf{g}$ T2 TSE. All images are axial. Asterisks in (d) and (g) mark areas defined as infiltration zone. N.B. these two groups differ in their IDH status and therefore this graph also depicts the IDH status differentiation potential of $\mathrm{K}^{\text {trans }}$ measured in vital high-grade glioma tissue 
this is already less established for proangiogenic factors, such as VEGF-A expression itself. In a study by Jensen et al., and confirmed by our results, $\mathrm{v}_{\mathrm{p}}$ was the only kinetic parameter that correlated to a significant degree with VEGF-A [2]. In contrast, in the study of Awasthi et al. VEGF-A expression was correlated exclusively with $k_{\text {ep }}$ [13] and in the study by Di et al. exclusively with $\mathrm{K}^{\text {trans }}$ [33], showing the low degree of data coherence. Di et al. could further only establish an association in vital tumor areas of high-grade glioma.

A strength of the present study is that multiple biopsies were taken in regions outside the apparent tumor and even in healthy tissue. Kinetic parameters mostly reflected microvascular parameters poorly outside the vital tumor. Limited detectability of BBBD with standard DCE-MRI is also a known obstacle in other areas such as dementia imaging [34]. As an exception, VEGF-A was associated more broadly with kinetic parameters in the infiltration zone than in vital tumor. The MVS was the only microvascular parameter showing association with kinetic parameters (here $\left.\mathrm{K}^{\text {trans}}\right)$ in the infiltration zone. These findings corroborate those of Tamura et al. regarding the unique features of VEGF expression and microvascularization of the infiltration zone in glioma [35].

Kinetic parameters did not differ between the vital tissue and infiltration zone of glioma of the same prognostic group but differed significantly between prognostic groups when comparing measurements in the same zones. As the IDH mutation status differed between prognostic groups 1 and 2, kinetic parameters could differentiate these when measured in both vital tumor and also infiltration zones, which is contradictory to findings by others for DCE-MRI $[16,18]$. It is noteworthy that despite not reaching a significant level of difference $\mathrm{K}^{\text {trans }}$ measured in healthy-rated tissue of prognostic groups 1 and 2 was minimally elevated compared to groups 3 and 4, which showed the expected virtual zero kinetic parameter values. This is an unexpected finding; however, the microscopically normally appearing biopsies came from regions, which were, after all, still very close to the tumor and not from distant locations. It might be possible that the BBB is already compromised at this point. The prognostically different groups 3 and 4 did not differ in radiological parameters. All of these findings imply that DCE-MRI has some potential to be used as a noninvasive advanced MRI method to assess gliomas beyond the vital tumor center, e.g., regarding the necessity for resection beyond the contrast-enhancing tumor boundaries. Furthermore, it stresses currently unsolved, but relevant questions regarding the reliability of kinetic parameter measurements in healthy brain tissue of glioma patients. The DCE-MRI sequences and post-processing adapted to low leakage may be a key solution.
However, in vital tumor tissue, kinetic parameters in this study also correlated with VAR and MVS, and more reliably than MVD. This finding corroborates the idea that large vessels and total vascularity of the examined tissue contribute more to the measurement than the sheer density of many small vessels. A similar finding was expressed very recently in a breast cancer model [36] and by Di et al. [19]. MVD differing significantly between prognostic groups supports that differences exist, but that DCE-MRI cannot show them. Notably, the parameter $v_{p}$ was not associated with any of the microvascular histological parameters in this study, despite being considered a substitute marker for vascularity and its high repeatability found even in healthy brain tissue [37]. Reasons for a limited comparability between results are, however, abundant in studies with quantitative MRI techniques. In the case of a $v_{p}$ analysis the arterial input function selection, e.g., can be an influential factor on results. At this point the choice of kinetic model needs to be addressed. While the extended Tofts model is generally not recommended for lesion analyses in low permeability regions, this compromise needed to be made for reasons of comparability of analyses between the tissue zones and histologically different lesions. The high correlations of presumably more robust model-free parameters, AUC and mrE, with the kinetic parameter $\mathrm{K}^{\text {trans }}$ also in infiltration zone and adjacent normal brain can be interpreted as an indicator that kinetic parameter measurements in these peripheral zones are still acceptably correct, albeit not ideal. This is supported by the work of Cramer et al., who showed a greater reliability for the Patlak model but a limited robustness also for the Tofts model, too, in low permeability tissues [23].

The large MR morphological differences in all parameters within corresponding regions and prognostic groups indicate a high variability of vascularization and VEGF-A expression. They also stress the problem of interindividual differences in kinetic parameters, also seen in this study, which hamper the establishment of cut-off values for lesion differentiation, and demanded a statistical approach balancing these differences in this study. It is possibly surprising that biopsies from contrast-enhancing regions of glioblastoma were evaluated as necrotic rather than vital tissue despite comparable kinetic values and careful handling of the tissue. An explanation could be a close proximity to necrosis in a region sometimes styled as the hypoxic penumbra of centrally necrotic glioma, which is an enhancing transition region to central necrosis [2].

\section{Limitations}

The numbers of patients and biopsies, especially in lower grade tumors, are limited and unevenly distributed. Only limited amounts of normal appearing brain tissue could be retrieved to avoid higher operative risk for patients. Another 
aspect is the clear separation of the different tumor areas and zones, which was based on classical neuroradiological criteria for this study; however, it could be assessed by (additive or alternative) artificial intelligence-based methods for future studies as are already available [38].

\section{Conclusion}

DCE-MRI seems sensitive for changes in the blood-brain barrier beyond the vital glioma tissue itself and may differentiate prognostically different gliomas in the infiltration zone and show an association with VEGF-A expression in this region.

Supplementary Information The online version of this article (https:// doi.org/10.1007/s00062-021-01015-3) contains supplementary material, which is available to authorized users.

Funding This study is university-funded.

\section{Declarations}

Conflict of interest V.C. Keil, G.H. Gielen, B. Pintea, P. Baumgarten, A. Datsi, K. Hittatiya,M. Simon and E. Hattingen declare that they have no competing interests.

Ethical standards All procedures performed in studies involving human participants or on human tissue were in accordance with the ethical standards of the institutional and/or national research committee and with the 1975 Helsinki declaration and its later amendments or comparable ethical standards. Ethics approval was given by the University Hospital Bonn ethics committee (file 358/13). All patients gave informed written consent prior to participation.

Open Access This article is licensed under a Creative Commons Attribution 4.0 International License, which permits use, sharing, adaptation, distribution and reproduction in any medium or format, as long as you give appropriate credit to the original author(s) and the source, provide a link to the Creative Commons licence, and indicate if changes were made. The images or other third party material in this article are included in the article's Creative Commons licence, unless indicated otherwise in a credit line to the material. If material is not included in the article's Creative Commons licence and your intended use is not permitted by statutory regulation or exceeds the permitted use, you will need to obtain permission directly from the copyright holder. To view a copy of this licence, visit http://creativecommons.org/licenses/by/4. $0 /$.

\section{References}

1. Khasraw M, Ameratunga MS, Grant R, Wheeler H, Pavlakis N. Antiangiogenic therapy for high-grade glioma. Cochrane Database Syst Rev. 2014;(9):CD008218. Update in: Cochrane Database Syst Rev. 2018;11:CD008218.

2. Jensen RL, Mumert ML, Gillespie DL, Kinney AY, Schabel MC, Salzman KL. Preoperative dynamic contrast-enhanced MRI correlates with molecular markers of hypoxia and vascularity in specific areas of intratumoral microenvironment and is predictive of patient outcome. Neuro Oncol. 2014;16:280-91.
3. Piludu F, Marzi S, Pace A, Villani V, Fabi A, Carapella CM, Terrenato I, Antenucci A, Vidiri A. Early biomarkers from dynamic contrast-enhanced magnetic resonance imaging to predict the response to antiangiogenic therapy in high-grade gliomas. Neuroradiology. 2015;57:1269-80.

4. Baker GJ, Yadav VN, Motsch S, Koschmann C, Calinescu AA, Mineharu Y, Camelo-Piragua SI, Orringer D, Bannykh S, Nichols WS, deCarvalho AC, Mikkelsen T, Castro MG, Lowenstein PR. Mechanisms of glioma formation: iterative perivascular glioma growth and invasion leads to tumor progression, VEGF-independent vascularization, and resistance to antiangiogenic therapy. Neoplasia. 2014;16:543-61.

5. Broermann A, Winderlich M, Block H, Frye M, Rossaint J, Zarbock A, Cagna G, Linnepe R, Schulte D, Nottebaum AF, Vestweber D. Dissociation of VE-PTP from VE-cadherin is required for leukocyte extravasation and for VEGF-induced vascular permeability in vivo. J Exp Med. 2011;208:2393-401.

6. Sottoriva A, Spiteri I, Piccirillo SG, Touloumis A, Collins VP, Marioni JC, Curtis C, Watts C, Tavaré S. Intratumor heterogeneity in human glioblastoma reflects cancer evolutionary dynamics. Proc Natl Acad Sci U S A. 2013;110:4009-14.

7. Dominietto M, Dobosz M, Bürgi S, Renner A, Zahlmann G, Scheuer W, Rudin M. Quantification of antiangiogenic treatment effects on tissue heterogeneity in glioma tumour xenograft model using a combination of DCE-MRI and 3D-ultramicroscopy. Eur Radiol. 2017;27:2894-902.

8. Blasel S, Franz K, Ackermann H, Weidauer S, Zanella F, Hattingen E. Stripe-like increase of rCBV beyond the visible border of glioblastomas: site of tumor infiltration growing after neurosurgery. J Neurooncol. 2011;103:575-84.

9. Juan-Albarracín J, Fuster-Garcia E, Pérez-Girbés A, Aparici-Robles F, Alberich-Bayarri Á, Revert-Ventura A, Martí-Bonmatí L, García-Gómez JM. Glioblastoma: Vascular Habitats Detected at Preoperative Dynamic Susceptibility-weighted Contrast-enhanced Perfusion MR Imaging Predict Survival. Radiology. 2018;287:94454.

10. Wu H, Tong H, Du X, Guo H, Ma Q, Zhang Y, Zhou X, Liu H, Wang S, Fang J, Zhang W. Vascular habitat analysis based on dynamic susceptibility contrast perfusion MRI predicts IDH mutation status and prognosis in high-grade gliomas. Eur Radiol. 2020;30:3254-65.

11. Tofts PS, Brix G, Buckley DL, Evelhoch JL, Henderson E, Knopp MV, Larsson HB, Lee TY, Mayr NA, Parker GJ, Port RE, Taylor J, Weisskoff RM. Estimating kinetic parameters from dynamic contrast-enhanced T(1)-weighted MRI of a diffusable tracer: standardized quantities and symbols. J Magn Reson Imaging. 1999;10:22332.

12. Xue W, Du X, Wu H, Liu H, Xie T, Tong H, Chen X, Guo Y, Zhang W. Aberrant glioblastoma neovascularization patterns and their correlation with DCE-MRI-derived parameters following temozolomide and bevacizumab treatment. Sci Rep. 2017;7:13894.

13. Awasthi R, Rathore RK, Soni P, Sahoo P, Awasthi A, Husain N, Behari S, Singh RK, Pandey CM, Gupta RK. Discriminant analysis to classify glioma grading using dynamic contrast-enhanced MRI and immunohistochemical markers. Neuroradiology. 2012;54:20513.

14. Choi HS, Kim AH, Ahn SS, Shin NY, Kim J, Lee SK. Glioma grading capability: comparisons among parameters from dynamic contrast-enhanced MRI and ADC value on DWI. Korean J Radiol. 2013;14:487-92.

15. Jia ZZ, Geng DY, Liu Y, Chen XR, Zhang J. Microvascular permeability of brain astrocytoma with contrast-enhanced magnetic resonance imaging: correlation analysis with histopathologic grade. Chin Med J (Engl). 2013;126:1953-6.

16. Ahn SS, Shin NY, Chang JH, Kim SH, Kim EH, Kim DW, Lee SK. Prediction of methylguanine methyltransferase promoter methylation in glioblastoma using dynamic contrast-enhanced 
magnetic resonance and diffusion tensor imaging. J Neurosurg. 2014;121:367-73.

17. Jia ZZ, Gu HM, Zhou XJ, Shi JL, Li MD, Zhou GF, Wu XH. The assessment of immature microvascular density in brain gliomas with dynamic contrast-enhanced magnetic resonance imaging. Eur J Radiol. 2015;84:1805-9.

18. Brendle C, Hempel JM, Schittenhelm J, Skardelly M, Tabatabai G, Bender B, Ernemann U, Klose U. Glioma Grading and Determination of IDH Mutation Status and ATRX loss by DCE and ASL Perfusion. Clin Neuroradiol. 2018;28:421-8.

19. Di N, Yao C, Cheng W, Ren Y, Qu J, Wang B, Yao Z. Correlation of dynamic contrast-enhanced MRI derived volume transfer constant with histological angiogenic markers in high-grade gliomas. J Med Imaging Radiat Oncol. 2018;62:464-70.

20. Lemée JM, Clavreul A, Aubry M, Com E, de Tayrac M, Eliat PA, Henry C, Rousseau A, Mosser J, Menei P. Characterizing the peritumoral brain zone in glioblastoma: a multidisciplinary analysis. $\mathrm{J}$ Neurooncol. 2015;122:53-61.

21. Artzi M, Liberman G, Blumenthal DT, Aizenstein O, Bokstein F, Ben Bashat D. Differentiation between vasogenic edema and infiltrative tumor in patients with high-grade gliomas using texture patch-based analysis. J Magn Reson Imaging. 2018;48:729-36.

22. van de Haar HJ, Burgmans S, Jansen JF, van Osch MJ, van Buchem MA, Muller M, Hofman PA, Verhey FR, Backes WH. Blood-Brain Barrier Leakage in Patients with Early Alzheimer Disease. Radiology 2016;281:527-35. Erratum in: Radiology. 2017;282:615.

23. Cramer SP, Larsson HB. Accurate determination of blood-brain barrier permeability using dynamic contrast-enhanced T1-weighted MRI: a simulation and in vivo study on healthy subjects and multiple sclerosis patients. J Cereb Blood Flow Metab. 2014;34:165565.

24. Louis DN, Perry A, Reifenberger G, von Deimling A, FigarellaBranger D, Cavenee WK, Ohgaki H, Wiestler OD, Kleihues P, Ellison DW. The 2016 World Health Organization Classification of Tumors of the Central Nervous System: a summary. Acta Neuropathol. 2016;131:803-20.

25. Hartmann C, Hentschel B, Wick W, Capper D, Felsberg J, Simon M, Westphal M, Schackert G, Meyermann R, Pietsch T, Reifenberger G, Weller M, Loeffler M, von Deimling A. Patients with IDH1 wild type anaplastic astrocytomas exhibit worse prognosis than IDH1-mutated glioblastomas, and IDH1 mutation status accounts for the unfavorable prognostic effect of higher age: implications for classification of gliomas. Acta Neuropathol. 2010;120:707-18.

26. Raica M, Cimpean AM, Anghel A. Immunohistochemical expression of vascular endothelial growth factor (VEGF) does not correlate with microvessel density in renal cell carcinoma. Neoplasma. 2007;54:278-84.

27. Baumgarten P, Brokinkel B, Zinke J, Zachskorn C, Ebel H, Albert FK, Stummer W, Plate KH, Harter PN, Hasselblatt M, Mittelbronn M. Expression of vascular endothelial growth factor (VEGF) and its receptors VEGFR1 and VEGFR2 in primary and recurrent WHO grade III meningiomas. Histol Histopathol. 2013;28:1157-66.

28. Baumgarten P, Blank AE, Franz K, Hattingen E, Dunst M, Zeiner P, Hoffmann K, Bähr O, Mäder L, Goeppert B, Machein M, Seifert V, Steinbach JP, Plate KH, Harter PN, Mittelbronn M. Differential expression of vascular endothelial growth factor A, its receptors VEGFR-1, -2, and -3 and co-receptors neuropilin-1 and -2 does not predict bevacizumab response in human astrocytomas. Neuro Oncol. 2016;18:173-83.

29. van Netten JP, Thornton IG, Carlyle SJ, Brigden ML, Coy P, Goodchild NL, Gallagher S, George EJ. Multiple microsample analysis of intratumor estrogen receptor distribution in breast cancers by a combined biochemical/immunohistochemical method. Eur J Cancer Clin Oncol. 1987;23:1337-42.

30. Kirkegaard T, Edwards J, Tovey S, McGlynn LM, Krishna SN, Mukherjee R, Tam L, Munro AF, Dunne B, Bartlett JM. Observer variation in immunohistochemical analysis of protein expression, time for a change? Histopathology. 2006;48:787-94.

31. Eichberg DG, Di L, Morell AA, Shah AH, Semonche AM, Chin CN, Bhatia RG, Jamshidi AM, Luther EM, Komotar RJ, Ivan ME. Incidence of high grade gliomas presenting as radiographically non-enhancing lesions: experience in 111 surgically treated non-enhancing gliomas with tissue diagnosis. J Neurooncol. 2020;147:671-9.

32. Heye AK, Culling RD, Valdés Hernández Mdel C, Thrippleton MJ, Wardlaw JM. Assessment of blood-brain barrier disruption using dynamic contrast-enhanced MRI. A systematic review. Neuroimage Clin. 2014;6:262-74.

33. Di N, Cheng W, Jiang X, Liu X, Zhou J, Xie Q, Chu Z, Chen H, Wang B. Can dynamic contrast-enhanced MRI evaluate VEGF expression in brain glioma? An MRI-guided stereotactic biopsy study. J Neuroradiol. 2019;46:186-92.

34. Raja R, Rosenberg GA, Caprihan A. MRI measurements of BloodBrain Barrier function in dementia: A review of recent studies. Neuropharmacology. 2018;134:259-71.

35. Tamura R, Ohara K, Sasaki H, Morimoto Y, Yoshida K, Toda M. Histopathological vascular investigation of the peritumoral brain zone of glioblastomas. J Neurooncol. 2018;136:233-41.

36. Kim E, Kim J, Maelandsmo GM, Johansen B, Moestue SA. Antiangiogenic therapy affects the relationship between tumor vascular structure and function: A correlation study between micro-computed tomography angiography and dynamic contrast enhanced MRI. Magn Reson Med. 2017;78:1513-22.

37. Artzi M, Liberman G, Blumenthal DT, Bokstein F, Aizenstein O, Ben Bashat D. Repeatability of dynamic contrast enhanced vp parameter in healthy subjects and patients with brain tumors. J Neurooncol. 2018;140:727-37.

38. Juan-Albarracín J, Fuster-Garcia E, García-Ferrando GA, GarcíaGómez JM. ONCOhabitats: A system for glioblastoma heterogeneity assessment through MRI. Int J Med Inform. 2019;128:53-61. 